\title{
A model for estimating the relation between the Hall to Pedersen conductance ratio and ground magnetic data derived from CHAMP satellite statistics
}

\author{
L. Juusola, O. Amm, K. Kauristie, and A. Viljanen \\ Finnish Meteorological Institute, Space Research Unit, P.O. Box 503, 00101, Finland \\ Received: 28 February 2006 - Revised: 7 March 2007 - Accepted: 20 March 2007 - Published: 29 March 2007
}

\begin{abstract}
The goal of this study is to find a way to statistically estimate the Hall to Pedersen conductance ratio $\alpha$ from ground magnetic data. We use vector magnetic data from the CHAMP satellite to derive this relation. $\alpha$ is attained from magnetic satellite data using the 1-D Spherical Elementary Current Systems (SECS). The ionospheric equivalent current density can either be computed from ground or satellite magnetic data. Under the required 1-D assumption, these two approaches are shown to be equal, which leads to the advantage that the statistics are not restricted to areas covered by ground data. Unlike other methods, using magnetic satellite measurements to determine $\alpha$ ensures reliable data over long time sequences. The statistical study, comprising over 6000 passes between $55^{\circ}$ and $76.5^{\circ}$ northern geomagnetic latitude during 2001 and 2002, is carried out employing data from the CHAMP satellite. The data are binned according to activity and season. In agreement with earlier studies, values between 1 and 3 are typically found for $\alpha$. Good compatibility is found, when $\alpha$ attained from CHAMP data is compared with EISCAT radar measurements. The results make it possible to estimate $\alpha$ from the east-west equivalent current density $J_{\phi}[\mathrm{A} / \mathrm{km}]: \alpha=2.07 /\left(36.54 /\left|J_{\phi}\right|+1\right)$ for $J_{\phi}<0$ (westward) and $\alpha=1.73 /\left(14.79 /\left|J_{\phi}\right|+1\right)$ for $J_{\phi}>0$ (eastward). Using the same data, statistics of ionospheric and field-aligned current densities as a function of geomagnetic latitude and MLT are included. These are binned with respect to activity, season and IMF $B_{Z}$ and $B_{Y}$. For the first time, all three current density components are simultaneously studied this way on a comparable spatial scale. With increasing activity, the enhancement and the equatorward expansion of the electrojets and the R1 and R2 currents is observed, and in the nightside, possible indications of a Cowling channel appear. During southward IMF $B_{Z}$, the electrojets and the R1 and R2 currents are stronger and clearer than during northward $B_{Z}$. IMF $B_{Y}$ affects the orientation of the pattern.
\end{abstract}

Correspondence to: L. Juusola

(liisa.juusola@fmi.fi)
Keywords. Ionosphere (Auroral ionosphere; Electric fields and currents; Ionosphere-magnetosphere interactions)

\section{Introduction}

Ionospheric conductances and current distributions, in addition to being important to ionospheric studies, also reflect the dynamics of the entire magnetosphere through its coupling to the ionosphere. Ground-based measurements provide an ionospheric projection of the magnetosphere along the geomagnetic field lines, which can be used, for instance, to place magnetospheric satellite observations in context within the large-scale magnetospheric structures, and to distinguish between spatial and temporal gradients in satellite data.

In order to discern the often weak ionospheric signatures of the magnetospheric processes in ground measurements, the data need to be further processed. The "method of characteristics" (Amm, 1995) is a technique for obtaining distributions of the ionospheric conductances and currents from ground magnetic data and ionospheric electric field data. In addition to these data, the method requires an estimate of the Hall to Pedersen conductance ratio. The goal of this study is to provide such an estimate based on ground magnetic field data. The studies by Lester and Davies (1996) and Davies and Lester (1999) using EISCAT (Folkestad et al., 1983) radar data have already suggested that although the Hall and Pedersen conductances themselves do not follow the eastwest directed current density, their ratio does, and could therefore be determined from the current density. To build up statistical relations between the two parameters, long time sequences of conductance ratio observations are needed. These are difficult to obtain from ground-based radars, but using magnetic field data from a satellite to determine the conductance ratio ensures reliable data over long time sequences.

Apart from its use with the method of characteristics, the precipitation-related part of the conductance ratio provides

Published by Copernicus GmbH on behalf of the European Geosciences Union. 


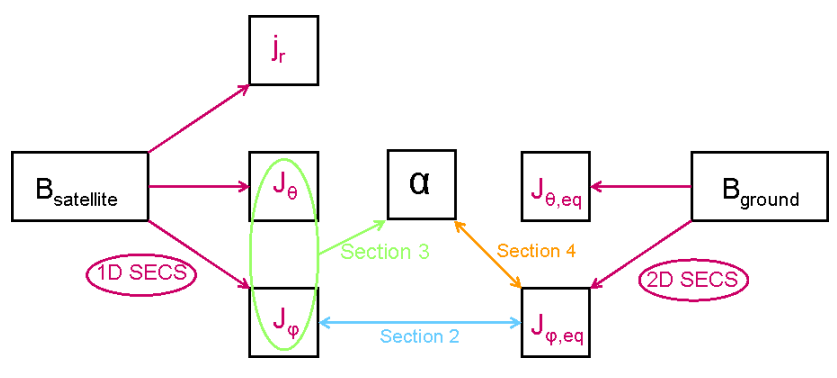

Fig. 1. The structure of the paper and the applied techniques. Section 2: Under the 1-D condition, $J_{\phi}$ determined from magnetic data from the CHAMP satellite using the 1-D SECS method is shown to be equal to $J_{\phi, e q}$, the $\phi$ component of the equivalent current density determined from IMAGE magnetometer data using the 2D SECS method ( $J_{\theta, e q}$ is the $\theta$ component of the equivalent current density). Section 3: Under the 1-D condition, $\alpha$ is shown to equal $-J_{\phi} / J_{\theta}$. Section 4: Magnetic vector data from the CHAMP satellite during 2001-2002 is used to make statistical maps of the ionospheric and field-aligned current density components, and to establish a relationship between $J_{\phi}$ and $\alpha$. This makes it possible to estimate $\alpha$ from ground magnetic data. In a 1-D case, $J_{\theta}=J_{c f}$ and $J_{\phi}=J_{d f} . j_{r}$ is the field-aligned component. In a 2-D case, $\boldsymbol{J}_{d f}=J_{\theta, e q} \hat{\boldsymbol{e}}_{\theta}+J_{\phi, e q} \hat{\boldsymbol{e}}_{\phi}$.

information on the altitude-dependence of the horizontal currents. In addition, it can be used to determine the characteristic energy of electron precipitation in the ionosphere (Robinson et al., 1987).

The ionospheric currents, like any vector field, can be expressed as a sum of divergence-free and curl-free components. Field-aligned currents (FAC) are then associated with the divergence of the curl-free component. For uniform conductances, the divergence-free and curl-free currents equal the Hall and Pedersen currents, respectively. Fukushima (1976) showed that for any 3-D current system, consisting of ionospheric and field-aligned currents, there exists an ionospheric sheet current distribution, called the equivalent currents, which causes the same magnetic field below the ionosphere as the original 3-D distribution. He also showed that the combined magnetic field of the curl-free currents and radial FACs is confined above the ionosphere. Therefore, this part of the field can only be measured by low-orbit satellites. The magnetic field caused by the divergence-free currents, on the other hand, can be attained either by ground or satellite measurements, and therefore the divergence-free currents equal the equivalent currents. A draw-back with data from only one satellite is that the current distribution has to be assumed both 1-D (independent of longitude in spherical geometry) and stationary during the satellite pass. This restricts useful data mostly to electrojet dominated cases.

In this study, the divergence-free ionospheric currents are used to represent the ground magnetic field. The Hall to Pedersen conductance ratio $(\alpha)$ is attained from the ratio of the divergence-free and curl-free current densities $\left(J_{d f} / J_{c f}\right)$. 1D Spherical Elementary Current Systems (SECS, Vanhamäki et al., 2003; Juusola et al., 2006) have been used to determine both $J_{d f}$ and $J_{c f}$ from magnetic data from the CHAMP (CHAllenging Minisatellite Payload; Ritter et al., 2004; http: //op.gfz-potsdam.de/champ/) satellite.

We begin by showing that in 1-D cases, the divergencefree currents determined from satellite data with the relatively new 1-D SECS method equal the equivalent currents obtained from ground measurements with the already wellestablished 2-D SECS method (Amm, 1997; Amm and Viljanen, 1999; Pulkkinen et al., 2003; Viljanen et al., 2004). For this purpose, we have used 124 1-D passes of the CHAMP satellite over the IMAGE (International Monitor for Auroral Geomagnetic Effects; Lühr et al., 1998; http://space.fmi.fi/ image/) magnetometer network during 2001 and 2002. In Sect. 3, the conditions under which $J_{d f} / J_{c f}$ can be used to represent $\alpha$ are discussed, and $J_{d f} / J_{c f}$ is compared to $\alpha$ measured by EISCAT.

Once the ability of the satellite-based $J_{d f}$ to represent the ground-based magnetic field and that of $J_{d f} / J_{c f}$ to represent $\alpha$ have been validated, useful data can be extended from the 124 IMAGE overpasses to 6112 1-D overpasses between $55^{\circ}$ and $76.5^{\circ}$ magnetic latitude during 2001 and 2002. In Sect. 4.3 , these data have then been used to establish a statistical estimate for $\alpha$ as a function of $J_{d f}$. The data have been binned according to the level of activity and season. In Sect. 4.2, we have included statistical maps of the ionospheric current densities and FACs during the two years as a function of MLT and geomagnetic latitude. Those have been binned with respect to activity, season and IMF $B_{Z}$ and $B_{Y}$. Fig. 1 illustrates graphically the structure of the paper and the applied techniques.

\section{Comparing ground- and satellite-based equivalent currents}

Throughout this paper we use a spherical coordinate system $(r, \theta, \phi)$ with its origin in the center of the Earth. The pole $(\theta=0)$, however, varies. In this section, for instance, it is chosen to coincide with the geomagnetic north pole at $79.5^{\circ}$ geographic latitude and $-71.6^{\circ}$ longitude. In a 1-D case, that is, when the current distribution is independent of $\phi$, the divergence-free ionospheric currents are then in the $\phi$ direction and the curl-free currents in the $\theta$ direction. The fieldaligned currents are assumed to flow radially.

When using the 1-D SECS method, the $r$ component of the magnetic field $\left(B_{r}\right)$, gives the $\phi$ component of the ionospheric current density $\left(J_{\phi}\right)$, while $B_{\phi}$ gives $J_{\theta}$ and the FACs. Vanhamäki et al. (2003) applied the 1-D SECS method to ground data and therefore determined $J_{\phi}$ by fitting $B_{\theta}$, which on ground is less anomalous than $B_{r}$. With satellite data, on the other hand, we use $B_{r}$, because FACs that in reality are never completely $1-\mathrm{D}$, affect $B_{\theta}$ while leaving $B_{r}$ practically 


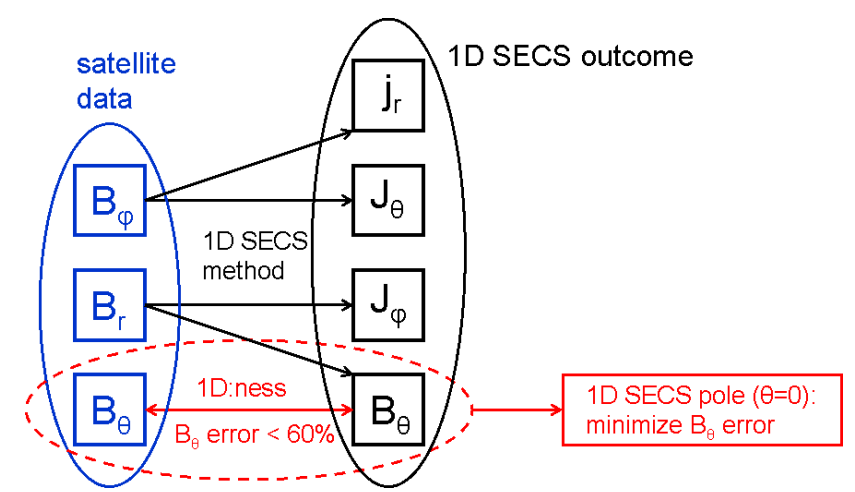

Fig. 2. Determining ionospheric currents from magnetic satellite data using the 1-D SECS method: $B_{\phi}$ gives $j_{r}$ and $J_{\theta}$, and $B_{r}$ gives $J_{\phi}$. 1-D:ness of the current distribution can be estimated from the difference between the 1-D SECS $B_{\theta}$, attained by fitting $B_{r}$, and that measured by the satellite. The optimal location for the 1-D SECS pole can be found by minimizing the $B_{\theta}$ error (Sect. 3.2).

intact. The difference between the 1-D SECS $B_{\theta}$ and that measured by the satellite can be used to determine how 1-D the current distribution is. Respective tests have shown that passes over $55^{\circ}-76.5^{\circ}$ latitude with an error

$\boldsymbol{B}_{\theta}$ error $=\frac{\overline{\left|\boldsymbol{B}_{\theta}{ }^{\text {measured }}-\boldsymbol{B}_{\theta}{ }^{1-\mathrm{D} \mathrm{SECS}}\right|}}{\overline{\left|\boldsymbol{B}_{\theta}{ }^{\text {measured }}\right|}} \times 100 \%$.

less than $60 \%$ are adequately $1-\mathrm{D}$ to give reliable results. $\overline{\left|\boldsymbol{B}_{\theta}\right|}$ stands for the average of the absolute values of the components of the vector. Figure 2 illustrates graphically the application of the 1-D SECS method to magnetic satellite data to obtain ionospheric and field-aligned currents and to determine the 1-D:ness of the current distribution.

Figure 3 shows the track of the satellite on 6 November at 05:04-05:08 UT, along with the schematic equivalent currents, attained by rotating the IMAGE ground horizontal magnetic field vectors $90^{\circ}$ clockwise. $B_{\theta}$ along the satellite track, as measured by CHAMP, is shown in the top left hand side panel of Fig. 4, along with the 1-D SECS $B_{\theta}$ attained by fitting $B_{r}$. The top right hand side panel displays similar data for 20 November 2001, at 03:59-04:04 UT. The first overflight is the same that was used by Juusola et al. (2006) as an example when first introducing the use of 1-D SECS to satellite data. The second one passed close to the EISCAT radar. In Sect. 3, we want to compare the Hall to Pedersen conductance ratio determined from CHAMP data with that determined from EISCAT data, and therefore this case has been selected as an example here and again later in Sect. 3.

In both cases, except for small scale variations, the measured $B_{\theta}$ and the $B_{\theta}$ resulting from the $B_{r}$ fit have very similar shapes, which indicates that both cases are adequately 1 D. In the lower panels of the figure are shown the divergencefree current density $J_{\phi}$ determined from CHAMP data using ground equivalent currents, 06-Nov-2001 05:05:00

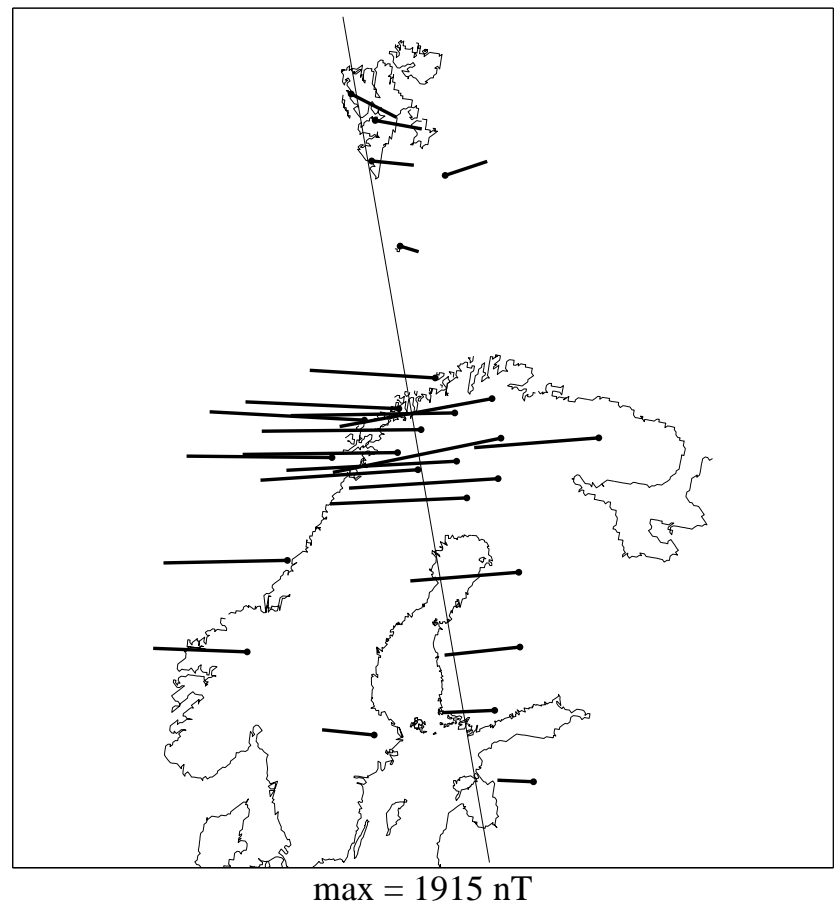

Fig. 3. Schematic equivalent currents, attained by rotating the IMAGE ground horizontal magnetic field vectors $90^{\circ}$ clockwise and the track of CHAMP on 6 November 2001, at 05:04-05:08 UT.

the 1-D SECS method and from IMAGE data using the 2-D SECS method. The current density determined from IMAGE data is averaged over the approximately four minutes it takes for the satellite to pass the network. Again the curves are very similar, as expected in a 1-D case.

A more comprehensive view is gained from Fig. 5, which shows a scatter plot of ground-based versus satellite-based $J_{\phi}$ for $B_{\theta}$ error $<60 \%$ (124 overpasses). Each point in the plot corresponds to one measurement in the $1 \mathrm{~Hz}$ CHAMP data. During the IMAGE overflight on 6 November 2001, for instance, the total number of measurement points was 275. Hence, the total number of points in the figure is approximately $124 \times 275$. A line fitted to the points using the least-squares method is shown in red, and the linear correlation coefficient is denoted by $r$. In blue is drawn a line passing through the origin with a unit slope.

Similar plots (not shown here) were also made for other degrees of 1-D:ness ( $B_{\theta}$ error $<80 \%$ : 435 passes; $>0 \%$ : 1073 passes; >80\%: 638 passes). The more 1-D the overflights are, the better correlation there is between the ground- and satellite-based $J_{\phi}$ (error $<60 \%$ : $r=0.90 ;<80 \%$ : $r=0.86$; all data: $r=0.82 ;>80 \%$ : $r=0.67)$. These results are in agreement with those attained by Ritter et al. (2004). They compared equivalent currents determined from CHAMP data using the method devised by Olsen (1996) with 

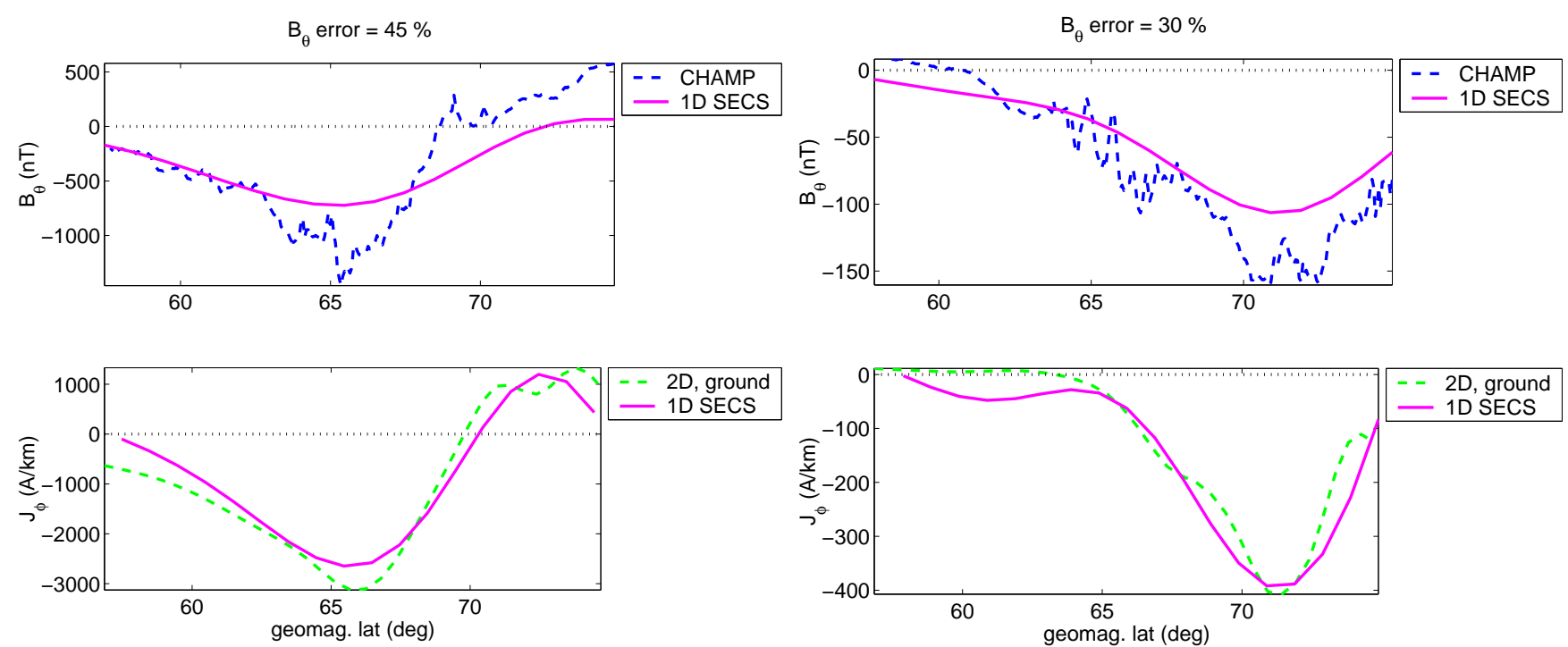

Fig. 4. Left: Top: $B_{\theta}$ measured by CHAMP on 6 November 2001 at 05:04-05:08 UT above IMAGE, and the 1-D SECS $B_{\theta}$ attained by fitting $B_{r}$. The similar shapes of the two curves (error $45 \%$ ) imply that the current distribution is reasonably 1-D during the overflight. Bottom: $J_{\phi}$ determined from CHAMP data by the 1-D SECS method and from IMAGE data by the 2-D SECS method. As expected in a 1-D case, the two profiles are almost the same. Right: The same as on the left hand side, but on 20 November 2001 at 03:59-04:04 UT.

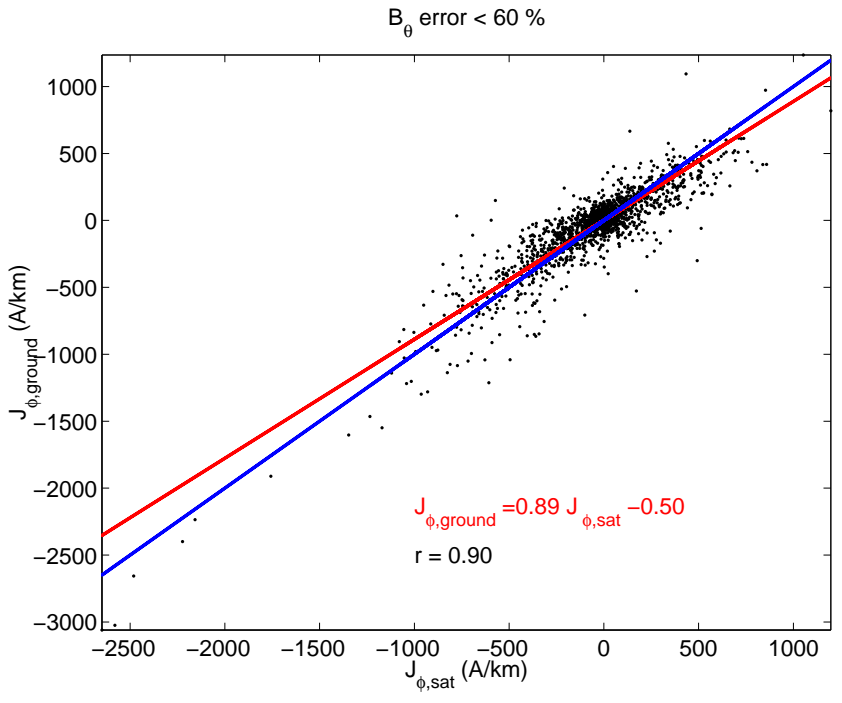

Fig. 5. Scatter plot of $J_{\phi}$ determined from ground-based measurements (IMAGE) by the 2-D SECS method with respect to $J_{\phi}$ determined from satellite-based measurements (CHAMP) by the 1-D SECS method. The ground-based $J_{\phi}$ is averaged over the approximately four minutes it takes for the satellite to pass over IMAGE. In red is shown a line fitted to the points, and the linear correlation coefficient is denoted by $r$. A line passing through the origin with a unit slope is drawn in blue. To create this plot, data from 124 satellite passes over IMAGE with an error smaller than $60 \%$ during 2001 and 2002 were used. those determined from IMAGE data using the 2-D SECS method. They found a high degree of correlation (correlation coefficient of 0.96 between $65^{\circ}$ and $70^{\circ}$ geographic latitude) between the results from the two methods, and conluded that this current component can indeed be determined reliably both from ground and satellite data.

When the error is approximately less than $60 \%$, groundand satellite-based $J_{\phi}$ can be considered to be equivalent. This means, in addition to confirming the reliability of the 1-D SECS method, that in our pursuit for an estimate for the conductance ratio as a function of ground-based magnetic data, we no longer need to consider the actual ground data at all, but instead can rely solely on CHAMP data with $J_{\phi}$ representing the ground data. Not being restricted to IMAGE passes extends the number of useful overflights in our statistical analysis of the following sections from 124 to 6112 (between $55^{\circ}$ and $76.5^{\circ}$ geomagnetic latitude).

\section{Determining the Hall to Pedersen conductance ratio}

\subsection{Relation between $\alpha$ and $-J_{\phi} / J_{\theta}$}

Assuming that the geomagnetic field is radial $\left(\boldsymbol{B}=-B \hat{\boldsymbol{e}}_{r}\right)$, and the convection electric field horizontal $\left(\boldsymbol{E}=E_{\theta} \hat{\boldsymbol{e}}_{\theta}+E_{\phi} \hat{\boldsymbol{e}}_{\phi}\right)$, Ohm's law in the ionosphere

$$
\boldsymbol{J}=\Sigma_{P} \boldsymbol{E}-\Sigma_{H} \frac{\boldsymbol{E} \times \boldsymbol{B}}{B}
$$


becomes

$$
\boldsymbol{J}=(\underbrace{\Sigma_{P} E_{\theta}+\Sigma_{H} E_{\phi}}_{=J_{\theta}}) \hat{\boldsymbol{e}}_{\theta}+(\underbrace{\Sigma_{P} E_{\phi}-\Sigma_{H} E_{\theta}}_{=J_{\phi}}) \hat{\boldsymbol{e}}_{\phi} .
$$

This gives the Hall to Pedersen conductance ratio in the Northern Hemisphere as

$\alpha=\frac{\Sigma_{H}}{\Sigma_{P}}=\frac{\frac{E_{\phi}}{E_{\theta}}+\left(-\frac{J_{\phi}}{J_{\theta}}\right)}{1-\left(-\frac{J_{\phi}}{J_{\theta}}\right) \cdot \frac{E_{\phi}}{E_{\theta}}}$,

which reduces to

$\alpha=-\frac{J_{\phi}}{J_{\theta}}$,

when $E_{\phi}=0$. It is obvious that if $E_{\phi} \neq 0,-J_{\phi} / J_{\theta}$ may differ significantly from $\alpha$. For example, if $-J_{\phi} / J_{\theta}=1$ and $E_{\phi} / E_{\theta}=0.3, \alpha \approx 2$. Therefore, it is clear that $-J_{\phi} / J_{\theta}$ can only be used to determine $\alpha$ if the direction of the convection electric field is known. Fortunately in 1-D cases, $E_{\phi} / E_{\theta} \approx 0$ is a good approximation: for uniform conductances, $J_{d f}=J_{H}$ and $J_{c f}=J_{P}$. On the other hand, in 1-D cases, $J_{d f}=J_{\phi}$ and $J_{c f}=J_{\theta}$ (Vanhamäki et al., 2003; Juusola et al., 2006). Combining these two gives $J_{\theta} \hat{\boldsymbol{e}}_{\theta}=\boldsymbol{J}_{P}=\Sigma_{P} \boldsymbol{E}$ and $J_{\phi} \hat{\boldsymbol{e}}_{\phi}=\boldsymbol{J}_{H}=-\Sigma_{H} \boldsymbol{E} \times \boldsymbol{B} / B$, which means that $\boldsymbol{E}=E \hat{\boldsymbol{e}}_{\theta}$. Although this result was attained by assuming uniform conductances in addition to 1-D:ness, it should be reasonably safe to state that in $1-\mathrm{D}$ cases, $E_{\phi} / E_{\theta} \approx 0$ and hence, $\alpha=-J_{\phi} / J_{\theta}$.

\subsection{Optimizing 1-D:ness}

The 1-D:ness of a certain current distribution depends on the location of the 1-D SECS pole. For instance, the 1-D:ness of a slightly tilted current distribution could be improved by moving the pole in such a way that in the new coordinate system the tilt disappears. In order to get the best possible results from our two years of CHAMP data, we optimized the location of the 1-D SECS pole for each overflight in the following way:

1. One satellite pass between $55^{\circ}$ and $76.5^{\circ}$ geomagnetic latitude, as defined in Sect. 2, was termed an "overflight". Most electrojets can be expected to fall within this latitude range.

2. The location of the 1-D SECS pole was restricted in such a way that in the new coordinates, the entire overflight fitted within $-77^{\circ} \ldots 77^{\circ}$ latitude, the 1-D SECS applicability region (Juusola et al., 2006), and the overflight spanned at least $10^{\circ}$ in latitude.

3. Starting from the geomagnetic pole, the optimal location for the 1-D SECS pole $\left(\theta_{0}, \phi_{0}\right)$ was found by minimizing the $B_{\theta}$ error (Eq. 1) using the Powell method (Press et al., 1992).
The Powell method can be used to minimize a function $f(\boldsymbol{P})$, where $\boldsymbol{P}$ is a point in $N$-dimensional space with unit vectors $\hat{\boldsymbol{e}}_{i}, i=1, \ldots, N$. The basic idea of the method is:

1. Start minimization at a point $\boldsymbol{P}_{0}$.

2. For $i=1, \ldots, N, \boldsymbol{P}_{i}=$ minimum along direction $\hat{\boldsymbol{e}}_{i}$, starting at $\boldsymbol{P}_{i-1}$.

3. The new value for $\boldsymbol{P}_{0}=$ minimum along direction $\boldsymbol{P}_{N}-\boldsymbol{P}_{0}$, starting at $\boldsymbol{P}_{N}$.

Steps 1-3 are repeated until $f$ stops decreasing.

Figure 6 shows a typical example of the optimization process for the overflight on 20 November 2001 at 03:5904:05 UT. The black line with time stamps shows the track of the CHAMP satellite with the 1-D optimized overflight highlighted in magenta. The color coding according to the color bar on the right hand side of the plot displays the $B_{\theta}$ error (Eq. 1) for the overflight with the 1-D SECS pole at each location. 1-D pole locations that are rejected due to the restriction that in the new coordinate system the overflight must fit within $-77^{\circ} \ldots 77^{\circ}$ 1-D latitude and span at least $10^{\circ}$ in latitude are automatically assigned an error of $100 \%$, which is why most of the plot appears reddish-brown. The yellow dot shows the starting-point for the optimization with the Powell method at the geomagnetic pole and the magenta dot the resulting $1-\mathrm{D}$ SECS pole at $79^{\circ}$ latitude and $-103^{\circ}$ longitude with a $B_{\theta}$ error of $23 \%$. The 1-D SECS pole is found in the dark blue minimum, which supports the suitability of the Powell method for the task. The bottom panel of Fig. 6 shows the steps taken during the Powell method optimization. The magnetic field fits and current densities for the new 1-D coordinate system are displayed in Fig. 7 (see Sect. 3.3 for details).

The optimization process resulted altogether in $61121-\mathrm{D}$ overflights $\left(B_{\theta}\right.$ error $<60 \%$, average $46 \%$, standard deviation $11 \%$ ). The upper left hand panel of Fig. 10 shows the number of data points as a function of geomagnetic latitude and MLT. Most of the overflights take place at dawn and dusk, which implies that the 1-D SECS method prefers electrojet dominated cases, as expected.

\subsection{Comparison with EISCAT}

Out of the 6112 1-D overflights, only five passed near EISCAT with $\alpha$ data available. The overflight of 20 November 2001 presented in the previous section was the one passing closest to the radar $(\sim 50 \mathrm{~km})$. Figure 7 displays the three components of the magnetic field $\left(B_{r}, B_{\theta}, B_{\phi}\right)$ and current density $\left(j_{r}, J_{\theta}, J_{\phi}\right)$ as a function of the 1-D latitude for the abovementioned overflight. The pole of the 1-D system is located at $79^{\circ}$ geographic latitude and $-103^{\circ}$ longitude. $J_{\phi}$ has been determined from $B_{r}$, and $j_{r}$ and $J_{\theta}$ from $B_{\phi}$. The 1-D SECS $B_{\theta}$ results from the $B_{r}$ fit, and has an error of $23 \%$ compared to the measured $B_{\theta}$, which implies excellent 1-D:ness. $-J_{\phi} / J_{\theta}$-based $\alpha$ and $\alpha$ determined from EISCAT 


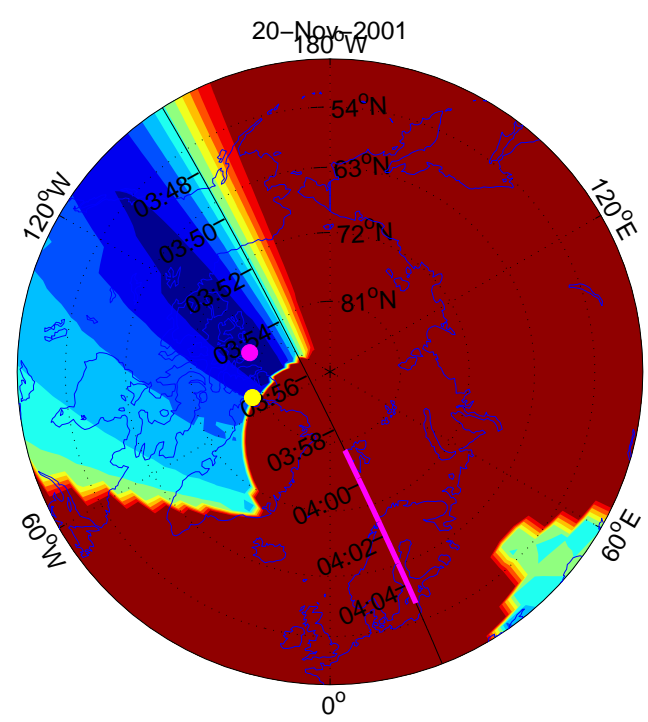

$\mathrm{B}_{\theta}$ error (\%)

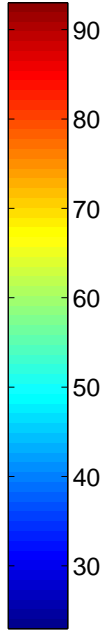

$\mathrm{B}_{\theta}$ error $(\%)$

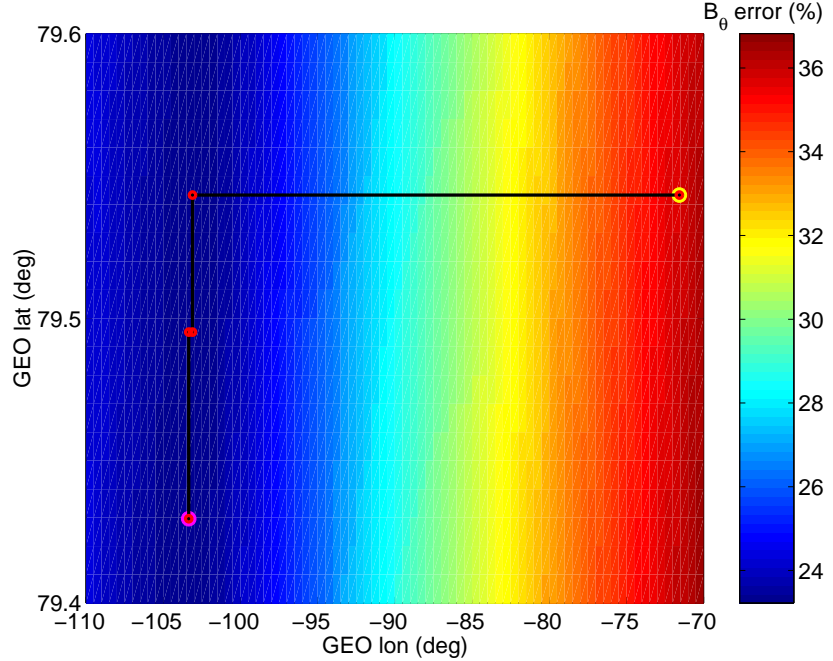

Fig. 6. Top: A typical example of the 1-D:ness optimization. The black line with the time stamps shows the track of the CHAMP satellite. The 1-D optimized part (overflight) is highlighted in magenta. The color coding according to the color bar on the right hand side of the plot displays the $B_{\theta}$ error (Eq. 1) for the overflight with the 1-D SECS pole at each location. The yellow dot shows the starting-point for the optimization at the geomagnetic pole and the magenta dot the resulting 1-D SECS pole at $79^{\circ}$ latitude and $-103^{\circ}$ longitude with $B_{\theta}$ error of $23 \%$. Bottom: A zoom in of the top panel showing also the steps taken during the optimization as red dots connected by a black line.

electron density measurements (with neutral collision frequencies from the MSIS-86 model, Hedin, 1987) as a function of geographic latitude are shown in the upper panel of Fig. 8. The lower panel illustrates the location of the satellite relative to the radar. Relative error of $-J_{\phi} / J_{\theta}$ and $\alpha$ above EISCAT is $26 \%$, which signifies a relatively good compatibility, when taking into account the different resolutions of
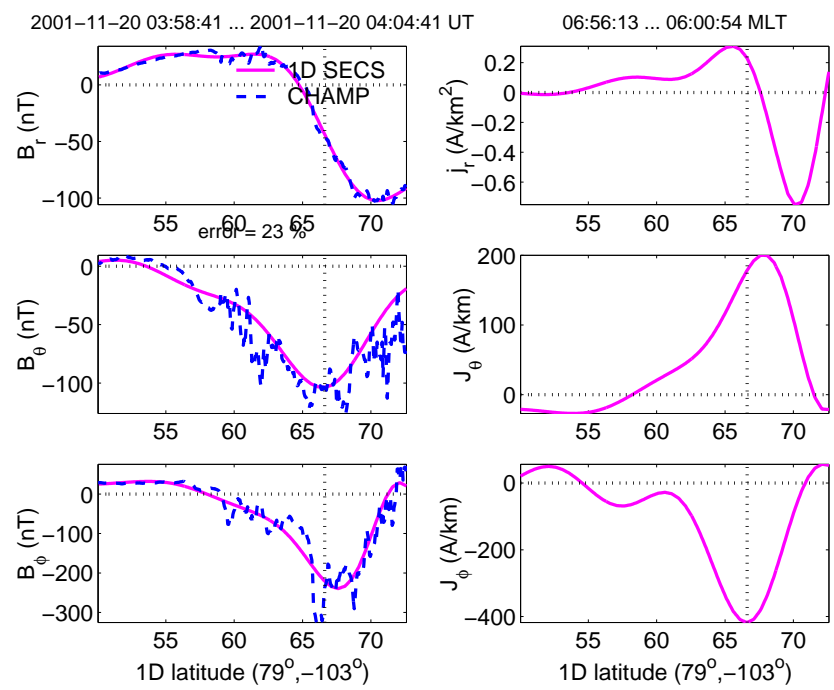

Fig. 7. The three components of the magnetic field $\left(B_{r}, B_{\theta}, B_{\phi}\right)$ and current density $\left(j_{r}, J_{\theta}, J_{\phi}\right)$ as a function of the 1-D latitude on 20 November 2001, at 03:59-04:04 UT. The pole of the 1-D system is located at $79^{\circ}$ latitude and $-103^{\circ}$ longitude. $J_{\phi}$ is determined from $B_{r}$, and $j_{r}$ and $J_{\theta}$ from $B_{\phi}$. The 1-D SECS $B_{\theta}$ results from the $B_{r}$ fit and has an error of $23 \%$.
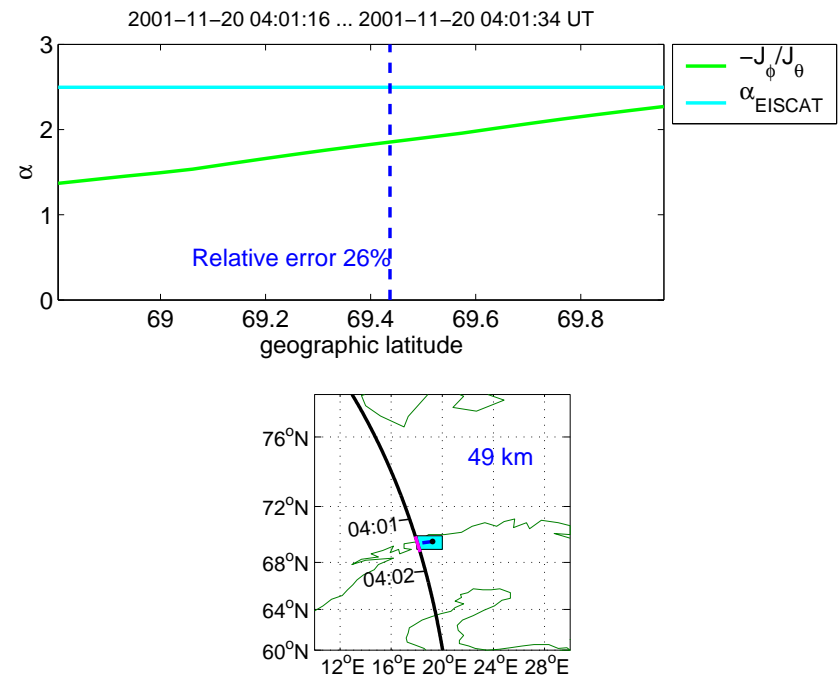

Fig. 8. Top: $-J_{\phi} / J_{\theta}$ and $\alpha$ measured by EISCAT as a function of geographic latitude. The vertical, blue, slashed line denotes the location of the radar. The relative error between the 1-D SECS and EISCAT $\alpha$ at that location is written in blue. The current densities and magnetic fields are displayed in Fig. 7. Bottom: The black curve denotes the track of the satellite, with the part displayed in the upper panel higlighted in magenta. The black dot in the cyan square denotes the location of the radar, and the blue line denotes the closest distance between the radar and the satellite. 


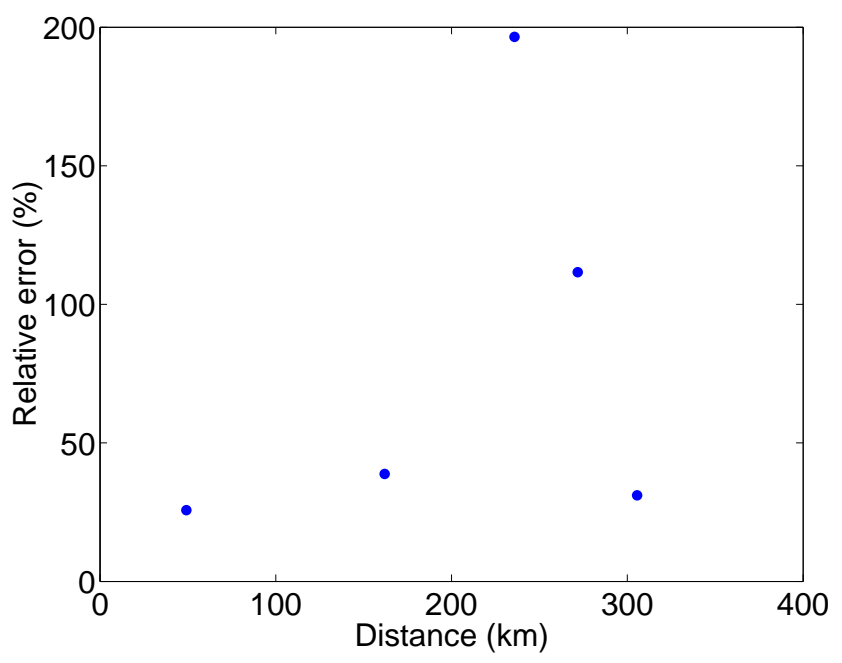

Fig. 9. Relative error between 1-D SECS and EISCAT $\alpha$ as a function of distance between the satellite and the radar for all five CHAMP overflights passing close to EISCAT with data from the radar available. From left to right, the times of the overflights are: 20 November 2001 04:01:16-04:01:34 UT, 9 October 2002 22:06:59-22:07:15 UT, 13 December 2001 02:14:02-02:14:20 UT, 6 October 2002 23:07:22-23:07:38 UT, and 23 November 2001 17:06:45-17:06:30 UT.

the two methods (1-D SECS: 150 km, Juusola et al. (2006); EISCAT: $\sim 1 \mathrm{~km}$, which is the width of the radar beam in the ionosphere), and the fact that the satellite did not pass directly above the radar. Figure 9 shows the relative error between 1-D SECS and EISCAT $\alpha$ for all the available cases as a function of distance between the satellite and the radar. The occurrence probability of equal alpha values as recorded by CHAMP and EISCAT should decrease with increasing distance. However, with the data set of only five points, we cannot appropriately demonstrate this trend. Figure 9 suggests that under favourable conditions, the two methods are indeed consistent with each other, with the relative error $<40 \%$. In two cases out of the total five, however, the two methods give completely different results. Resolving the reasons for these discrepancies (besides the abovementioned differences in spatial resolution) would require a more thorough EISCAT-CHAMP comparison study with a larger data set, which could be a topic for a future study.

The results of this section, combined with those of Sect. 3.1, indicate that in 1-D cases, using $-J_{\phi} / J_{\theta}$ for $\alpha$ should be a good approximation.
Table 1. The number of 1-D overfligts between $55^{\circ}$ and $76.5^{\circ}$ geomagnetic latitude during 2001 and 2002 with $\left|I_{\phi}\right|=0 \ldots 0.15 \mathrm{MA}$ (quiet), $\left|I_{\phi}\right|=0.15 \ldots 0.30 \mathrm{MA}$ (moderate), $\left|I_{\phi}\right|>0.30 \mathrm{MA}$ (active), as well as the number of overflights for each season (winter: January, February, November, December; equinox: March, April, September, October; summer: May, June, July, August).

\begin{tabular}{ll}
\hline & overflights \\
\hline Quiet & 2117 \\
Moderate & 1914 \\
Active & 2081 \\
Winter & 1733 \\
Equinox & 1938 \\
Summer & 2441 \\
\hline
\end{tabular}

\section{Statistical study}

\subsection{Data overview}

For this study, we have employed altogether 6112 1-D $\left(B_{\theta}\right.$ error $<60 \%$ ) overflights of CHAMP between $55^{\circ}$ and $76.5^{\circ}$ geomagnetic latitude in the Northern Hemisphere. The data have been binned according to the level of activity, represented by the absolute value of the total current in the $\phi$ direction $\left(\left|I_{\phi}\right|\right)$, and season. It should be noted that both sunlight and geomagnetic activity can increase $\left|I_{\phi}\right|$ and that, naturally, the effect of sunlight on $\left|I_{\phi}\right|$ is strongest on the dayside. We have divided the year into three seasons: winter (January, February, November, December), summer (May, June, July, August) and equinox (March, April, September, October).

Table 1 shows how many overflights fall into each category. The different activity ranges (quiet: $\left|I_{\phi}\right|=0 \ldots 0.15 \mathrm{MA}$, moderate: $\left|I_{\phi}\right|=0.15 \ldots 0.30 \mathrm{MA}$, active: $\left|I_{\phi}\right|>0.30 \mathrm{MA}$ ) were chosen in such a way that an approximately equal number of overflights would fall into each one. However, since the 1-D assumption restricts the data mainly to electrojet cases, they are all relatively quiet or moderately disturbed. For comparison, an infinitely long line current of $0.15 \mathrm{MA}$ at $100 \mathrm{~km}$ altitude would cause a magnetic field of $300 \mathrm{nT}$ on ground directly below, and a line current of 0.3 MA a magnetic field of $600 \mathrm{nT}$.

\subsection{Maps of $J_{\phi}, J_{\theta}$ and FACs}

Before going to $\alpha$-statistics, we have included in this section plots of average $j_{r}, J_{\theta}$ and $J_{\phi}$ as a function of geomagnetic latitude and MLT during the 6112 overpasses. To our knowledge, this is the first time that all three components are simultaneously studied this way on a comparable spatial scale. Due to the distance between the satellite and the ionosphere $(\sim 300 \mathrm{~km})$, the spatial resolution of $J_{\phi}$, which is not associated with FACs, is limited to $\sim 150 \mathrm{~km}$. In order to be able to compare the different current components meaningfully, and 

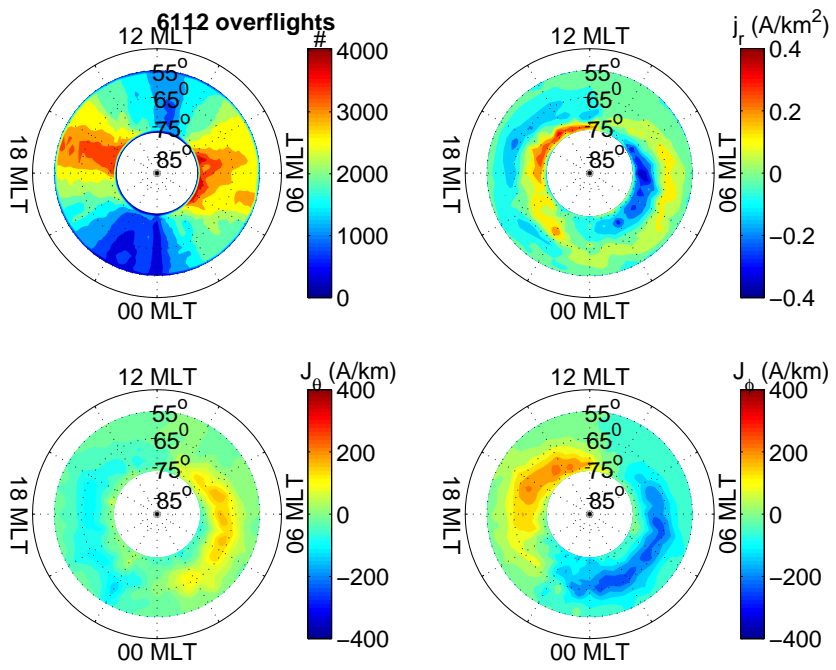

Fig. 10. Distribution of data points (top, left) for the 6112 1-D overflights as a function of geomagnetic latitude and MLT. Here, one data point refers to one measurement in the $1 \mathrm{~Hz}$ CHAMP data, with the total number of points 2195327. $j_{r}$ (top, right: field-aligned component, positive up), $J_{\theta}$ (bottom, left: north-south component, positive south) and $J_{\phi}$ (bottom, right: east-west component, positive east) as a function of MLT and magnetic latitude. The number of data points or the magnitude and direction of the current density in each cell is given according to the color bar on the right hand side of the plot. Resolution of the plots is $0.5 \mathrm{~h}$ in MLT and $1^{\circ}$ in latitude. On the top of the figure is written the total number of overpasses used to construct the set of four plots.

to compute $\alpha=-J_{\phi} / J_{\theta}$, also $J_{\theta}$ and FACs are determined at the same scale. This is accomplished by placing the 1-D SECSs $0.5^{\circ}$ apart in the ionosphere. As a result, the small scale components in the measured magnetic field in Fig. 7 (blue, slashed line) are not reproduced in the 1-D SECS fits and resulting current densities (magenta line).

Figure 10 shows the distribution of data points (one data point refers to one measurement in the $1 \mathrm{~Hz}$ CHAMP data), the average $j_{r}$ (field-aligned component, positive up), $J_{\theta}$ (north-south component, positive south) and $J_{\phi}$ (east-west component, positive east) as a function of geomagnetic latitude and MLT. The plot is constructed out of cells with dimensions of $0.5 \mathrm{~h}$ in MLT and $1^{\circ}$ in latitude, and the number of data points or the magnitude and direction of the currents in each cell is given according to the color bar on the right hand side of the plot. On the top of the figure is written the total number of overpasses used to construct the set.

As expected due to the 1-D assumption, most data points are concentrated in the electrojet dominated regions at dawn and dusk, and thus the reliablity of the current density plots is also best in these regions. The number of data points at noon and midnight is about $1 / 10$ of the number of points at dawn and dusk, suggesting that the statistical error of $j_{r}, J_{\theta}$ and $J_{\phi}$ at noon and midnight is about $\sqrt{10} \approx 3$ times larger than at dawn and dusk. The field-aligned currents display the typical pattern of Region 1 and Region 2 currents (Iijima and Potemra, 1976), with the amplitude of the poleward Region 1 currents clearly stronger than that of the equatorward Region 2 currents. On the nightside the upward (yellow and red) Region 1 currents are connected to the upward Region 2 currents in a configuration typical for the Harang discontinuity (Untiedt and Baumjohann, 1993). Of the horizontal currents, $J_{\theta}$ mainly corresponds to the curl-free currents and therefore connects the upward and downward field-aligned currents. $J_{\phi}$, on the other hand, mostly corresponds to the divergencefree (equivalent) currents. The distribution shows the typical eastward and westward electrojets located between the Region 1 and 2 field-aligned currents.

Figure 11 shows the data binned with respect to activity and season. The bin and the number of overpasses used to construct the four plots are denoted on top of each set. With increasing activity, the enhancement of the amplitude and the expansion of the pattern toward the equator are clearly visible. On the nightside, on the other hand, a more interesting feature occurs. With increasing activity, the westward electrojet (blue) appears to penetrate deeper into the evening side, while there are no corresponding changes in $J_{\theta}$. This might be an indication of a Cowling channel (Baumjohann et al., 1981). In a Cowling channel, the total current is westward directed, not just the Hall current. The penetration of $J_{\phi}$ might also be explained by non-zero IMF $B_{Y}$, but Fig. 12 indicates that in such a case there should also be a corresponding change in $J_{\theta}$. The binning with respect to season shows that in the post-noon sector $J_{\phi}$ and $j_{r}$ become stronger from winter to equinox to summer. This could be caused by sunlight, but since there is no corresponding enhancement to be seen on the morning side, it seems more likely that the effect is at least partly due to relatively strong cusp-related currents. In agreement with Weimer (2001), the winter patterns are clearly weaker than those during equinox or summer. According to Russel and McPherron (1973), an enhancement in magnetic activity is expected during equinox. In agreement with Ritter et al. (2004), the effect appears to be mostly confined in the nightside.

In Fig. 12 the data are binned with respect to IMF $B_{Z}$ and $B_{Y}$. There are three bins for negative $(<-3 \mathrm{nT})$, around zero $(<|3| \mathrm{nT})$ and positive $(>3 \mathrm{nT})$ values of both components. For negative $B_{Z}$, the basic pattern is clearer and stronger than for zero or positive $B_{Z}$, in agreement with the results of $\mathrm{Pa}$ pitashvili et al. (2002). For northward IMF the convection pattern is in general more complex than the basic two-cell configuration (e.g. Schunk and Nagy, 2000), which is dominant for southward IMF. Averaging over all these cases might be the cause for the rather faint and broken pattern in the plot, in addition to the relatively low number of data points used to construct this plot. The effect of binning with respect to IMF $B_{Y}$ shows most clearly in the FAC plots. On the dayside, the strongest Region 1 currents form an arc centered at 13:00 MLT for $B_{Y}<-3 \mathrm{nT}$, at 09:00 MLT for $\left|B_{Y}\right|<3 \mathrm{nT}$, 


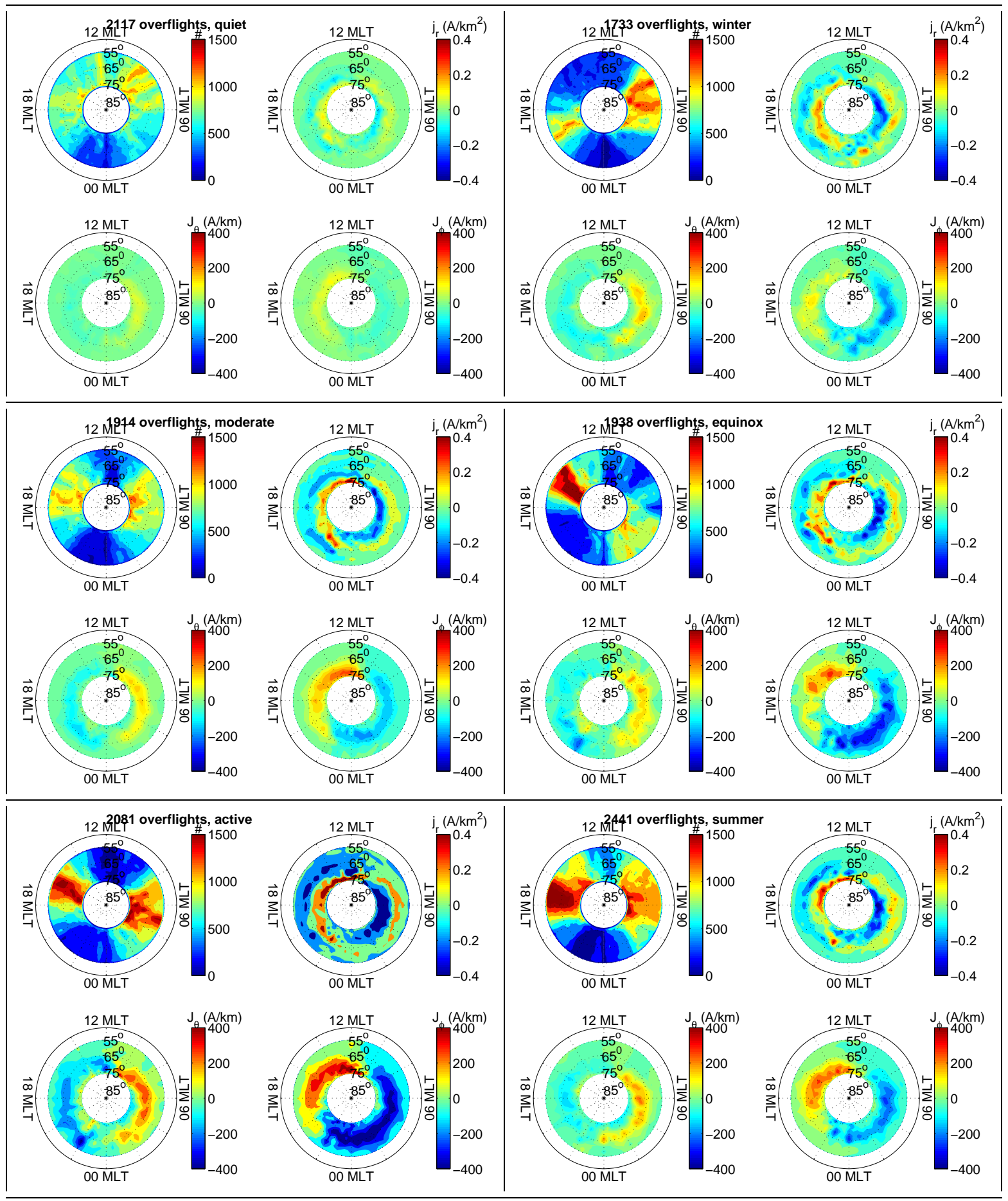

Fig. 11. The same as Fig. 10 except that the data are binned with respect to activity and season. The bin and the number of overpasses used to construct the four plots are denoted on top of each set. The plots in the left hand side column show the binning with respect to activity (quiet: $\left|I_{\phi}\right|<0.15 \mathrm{MA}$, moderate: $0.15 \leq\left|I_{\phi}\right|<0.30 \mathrm{MA}$, and active: $\left|I_{\phi}\right| \geq 0.30 \mathrm{MA}$ ) and the plots in the right hand side column with respect to season (winter, equinox, and summer). 


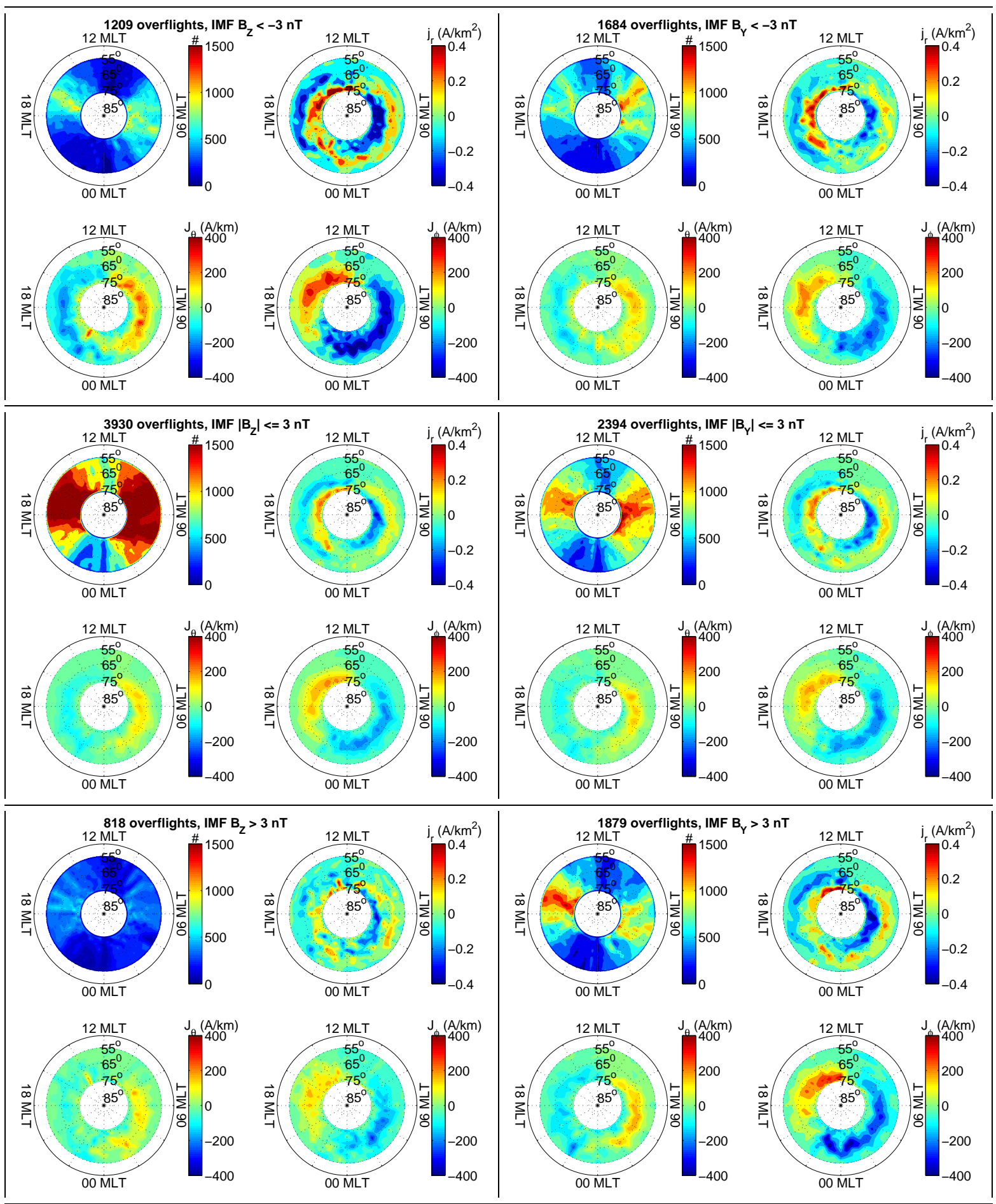

Fig. 12. The same as Fig. 10, except that the data are binned with respect to the IMF $B_{Z}$ and $B_{Y}$. There are bins for negative $(<-3 \mathrm{nT})$, around zero $(<|3| \mathrm{nT})$ and positive $(>3 \mathrm{nT})$ values of both components. The bin and the number of overpasses used to construct the four plots are denoted on top of each set. 
and at 08:00 MLT for $B_{Y}>3 \mathrm{nT}$. In $J_{\phi}$ plots, westward current (blue) on nightside ends at $\sim 24: 00$ MLT for $B_{Y}<-3 \mathrm{nT}$, at $\sim 23: 00$ MLT for $\left|B_{Y}\right|<3 \mathrm{nT}$, and at $\sim 22: 00$ MLT for $B_{Y}>3 \mathrm{nT}$.

\subsection{Statistical estimate for $\alpha$ as a function of $J_{\phi}$}

Our goal is to find a way to estimate $\alpha$ from the east-west current density, which can be determined from ground-based magnetic data. To accomplish that, we display the occurrence probability of different values of $\alpha=-J_{\phi} / J_{\theta}$ as a function of $J_{\phi}$ in the four panels in the left hand side column of Fig. 13. Summing up each column in one plot gives $100 \%$. For instance, if a westward current of $500 \mathrm{~A} / \mathrm{km}$ is measured, the probability of having $\alpha=2$, without specifying the activity conditions or season, can be determined from the left hand side branch of the top left hand side plot. The resolution of the plots is $25 \mathrm{~A} / \mathrm{km}$ in $J_{\phi}$ and 0.1 in $\alpha$. The different panels of the figure correspond to the different levels of activity: $0 \mathrm{MA} \leq\left|I_{\phi}\right|$ (top), $0 \mathrm{MA} \leq\left|I_{\phi}\right|<0.15 \mathrm{MA}$ (second from the top), $0.15 \mathrm{MA} \leq\left|I_{\phi}\right|<0.30 \mathrm{MA}$ (second from the bottom), $0.30 \mathrm{MA} \leq\left|I_{\phi}\right|$ (bottom). The four panels in the right hand side column of the figure are similarly arranged. It should be noted here that we are not separating substorm events from non-substorm events. The points in the figures represent the median, and the errorbars the 15.9 and 84.1 percentiles, calculated from the left hand side panels. Using the least-squares method, to the points is fitted the curve

$\alpha=-\frac{J_{\phi}}{J_{\theta}}=\frac{C_{2}}{\frac{C_{1}}{\left|J_{\phi}\right|}-1}$,

which corresponds to

$J_{\phi}=C_{1}+C_{2} J_{\theta}$,

where $C_{1}$ and $C_{2}$ are constants estimated in the fitting process. Whereas Eq. (5) suggests that $C_{2}$ alone should be enough to describe the relationship between the two current density components, $C_{1}$ is needed in Eq. (6) to correctly describe the behavior of $\alpha$ in the region where $\left|J_{\phi}\right|$ is small. The curves have been fitted separately in the regions where $J_{\phi}<0$ and $J_{\phi}>0$. The constants $C_{1}$ and $C_{2}$ resulting from the fit along with the residual $(r)$ are denoted in the plots. The same curves have also been included in the four left hand side panels of the figure. With increasing $\left|J_{\phi}\right|, \alpha$ approaches $-C_{2}$, and when $J_{\theta} \approx 0, J_{\phi} \approx C_{1}$. For small $\left|J_{\phi}\right|,\left|J_{\theta}\right|$ gets small as well. Figure 14 shows the data binned according to season.

In both figures, there is a clear asymmetry of $\alpha$ with respect to $J_{\phi}$. For $J_{\phi}>0$, corresponding mainly to the eastward electrojet, $\alpha$ is in general a little lower and less scattered than for $J_{\phi}<0$ (on the right hand side branch in the color panels, the $\alpha$ values are more concentrated (red), than on the left hand side branch (green and yellow)). This indicates an asymmetry in the electron precipitation in the dusk and dawn sectors of the auroral oval. The asymmetry is largest in the
Table 2. Coefficients $C_{1}$ and $C_{2}$ resulting from the fitting of Eq. (6) into the data displayed in Figs. 13 and 14.

\begin{tabular}{lllll}
\hline Bin & $C_{1}\left(J_{\phi}<0\right)$ & $C_{2}\left(J_{\phi}<0\right)$ & $C_{1}\left(J_{\phi}>0\right)$ & $C_{2}\left(J_{\phi}>0\right)$ \\
\hline All & -36.54 & -2.07 & -14.79 & -1.73 \\
Quiet & -20.35 & -2.19 & -46.16 & -2.53 \\
Moderate & -21.68 & -1.95 & -19.35 & -1.84 \\
Active & -80.63 & -2.22 & -26.05 & -1.74 \\
Winter & -21.87 & -1.99 & -13.79 & -1.42 \\
Equinox & -49.36 & -2.10 & -16.55 & -1.63 \\
Summer & -18.64 & -1.86 & -5.88 & -1.76 \\
\hline
\end{tabular}

winter, becoming smaller during the equinox and almost disappearing in the summer. In all cases, the fitted curve follows closely the data points, giving a simple means for approximating $\alpha$ once the equivalent current density is known. The fitted coefficients $C_{1}$ and $C_{2}$ from Figs. 13 and 14 are summarised in Table 2.

Schlegel (1988) used two years of EISCAT data (19851986) to compute histograms of the Hall to Pedersen conductance ratio. He found values between 0.25 and more than 4 for $\alpha$, with a mean of 1.64. The values for $\alpha$ in the uppermost left panel of Fig. 13 (mainly between 1 and 3) appear consistent with these results. He also determined that the mean value of $\alpha$ grows with increasing activity $\left(K_{p}\right)$. Although the curves resulting from the binning with respect to activity in Fig. 13 do not differ significantly, our results agree with his, since $J_{\phi}$, and therefore also $\alpha$, gets on average higher with increasing activity. In the dawn side, Schlegel's histograms are more spread out and with a higher mean value $(\sim 1.9$ at 06:00 MLT) than in the dusk sector ( 1.2 at 18:00 MLT). Our results are also consistent with these.

Davies and Lester (1999) calculated median values for $\alpha$ as a function of MLT. Between 18:00 and midnight they obtained $\alpha \approx 1.8$, whereas after midnight $\alpha$ was larger, at times exceeding 2 . Our results are in good agreement with these findings.

As an application, Fig. 15 illustrates distributions of $\alpha$, derived from the east-west component of the divergence-free or equivalent current density using Eq. (6) and the constants displayed in Table 2. In the uppermost plot, all data are incorporated, in the plots in the middle row, the data are binned with respect to activity, and in the plots in the bottom row, with respect to season (the bin is denoted on the left hand side of each plot). The colorbar on the right hand side of each plot gives the scaling. During low activity, $\alpha$ is also low. Increasing activity raises the conductance ratio especially around midnight and at dawn. Since activity is determined using the total current, part of the effects brought along with its increase, especially on dayside, are actually due to increased sunlight, not real geomagnetic activity. In winter, the conductance ratio is quite low, only on the dawn side there are slightly higher values. This is probably caused by substorm 

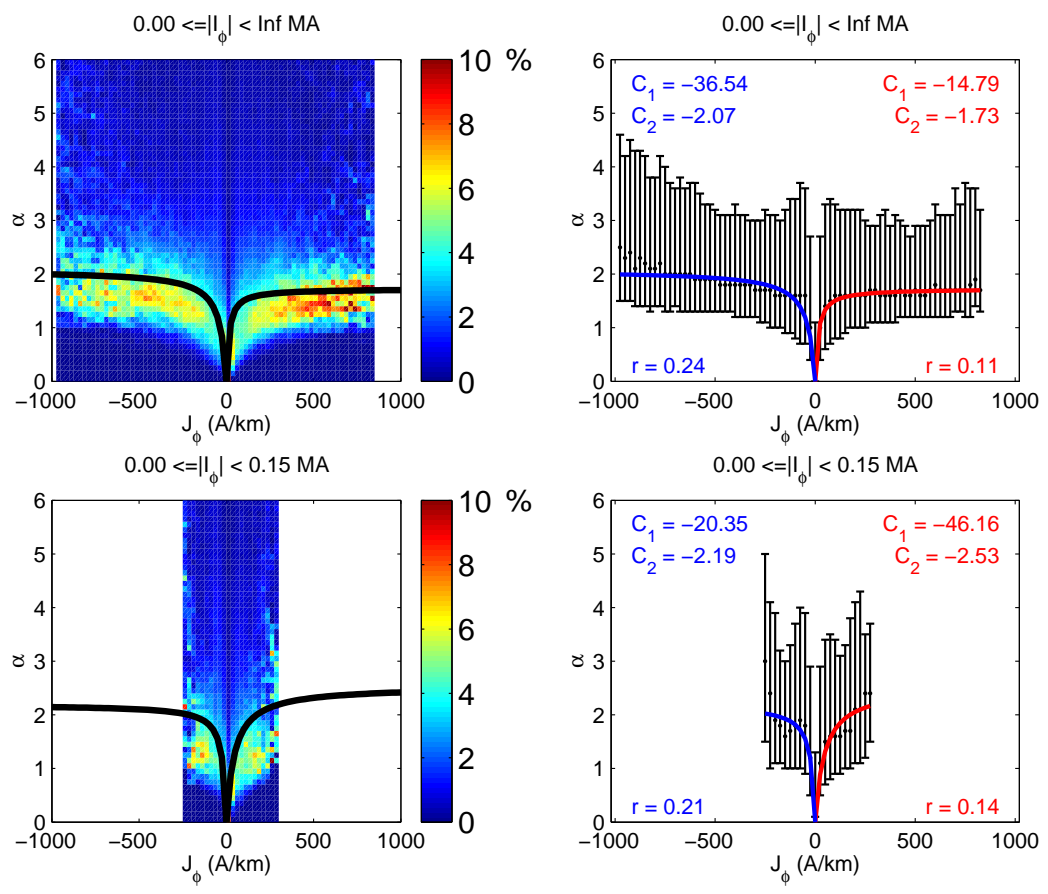

$0.15<=\left|I_{\phi}\right|<0.30 \mathrm{MA}$
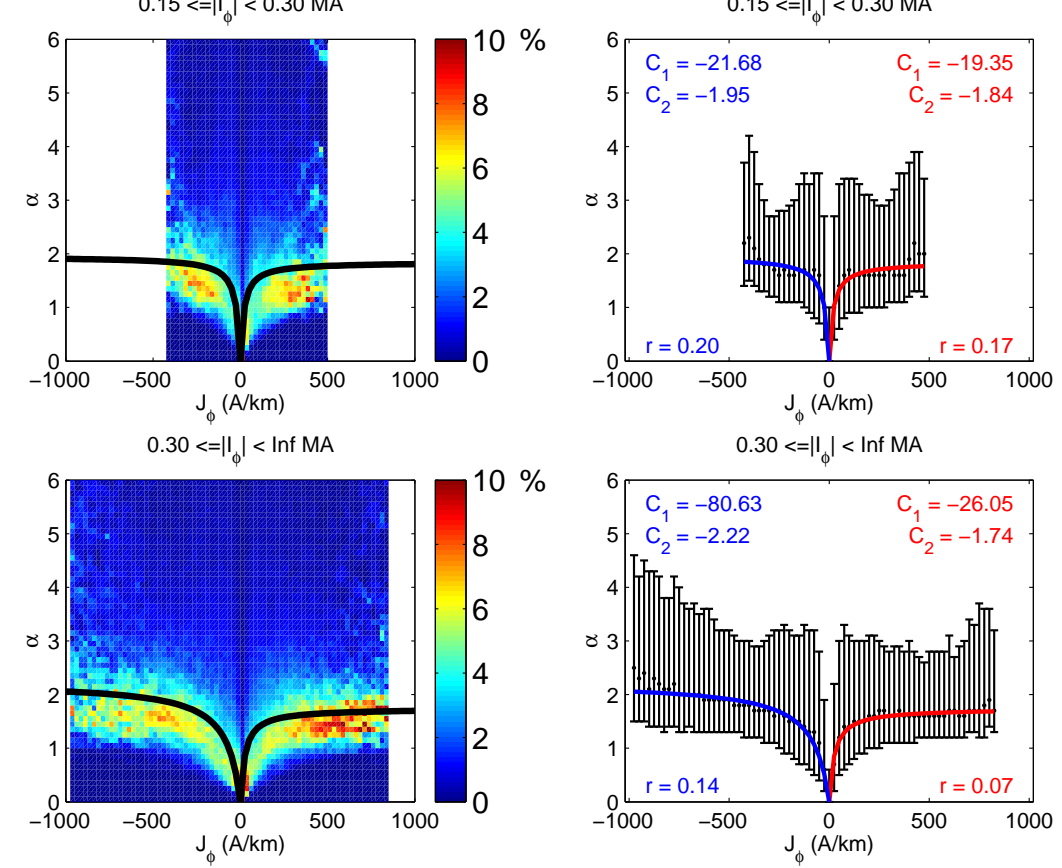

Fig. 13. Left: The probability for different values of $\alpha=-J_{\phi} / J_{\theta}$ as a function of $J_{\phi}$ (summing up each column gives $100 \%$ ). Columns with less than 500 data points are rejected (white). The resolution of the plots is $25 \mathrm{~A} / \mathrm{km}$ in $J_{\phi}$ and 0.1 in $\alpha$. The different panels correspond to the different levels of activity: $0 \mathrm{MA} \leq\left|I_{\phi}\right|$ (top), $0 \mathrm{MA} \leq\left|I_{\phi}\right|<0.15 \mathrm{MA}$ (second from top), $0.15 \mathrm{MA} \leq\left|I_{\phi}\right|<0.30 \mathrm{MA}$ (second from bottom), $0.30 \mathrm{MA} \leq\left|I_{\phi}\right|$ (bottom). Right: The four panels on the right hand side column of the figure are arranged similar to those on the left hand side. The points represent the median, and the errorbars the 15.9 and 84.1 percentiles. Using the least-squares method, to the points is fitted the curve of Eq. (6). The resulting constants $C_{1}$ and $C_{2}$ are denoted in the plots along with the residual $r$ of the fit. 

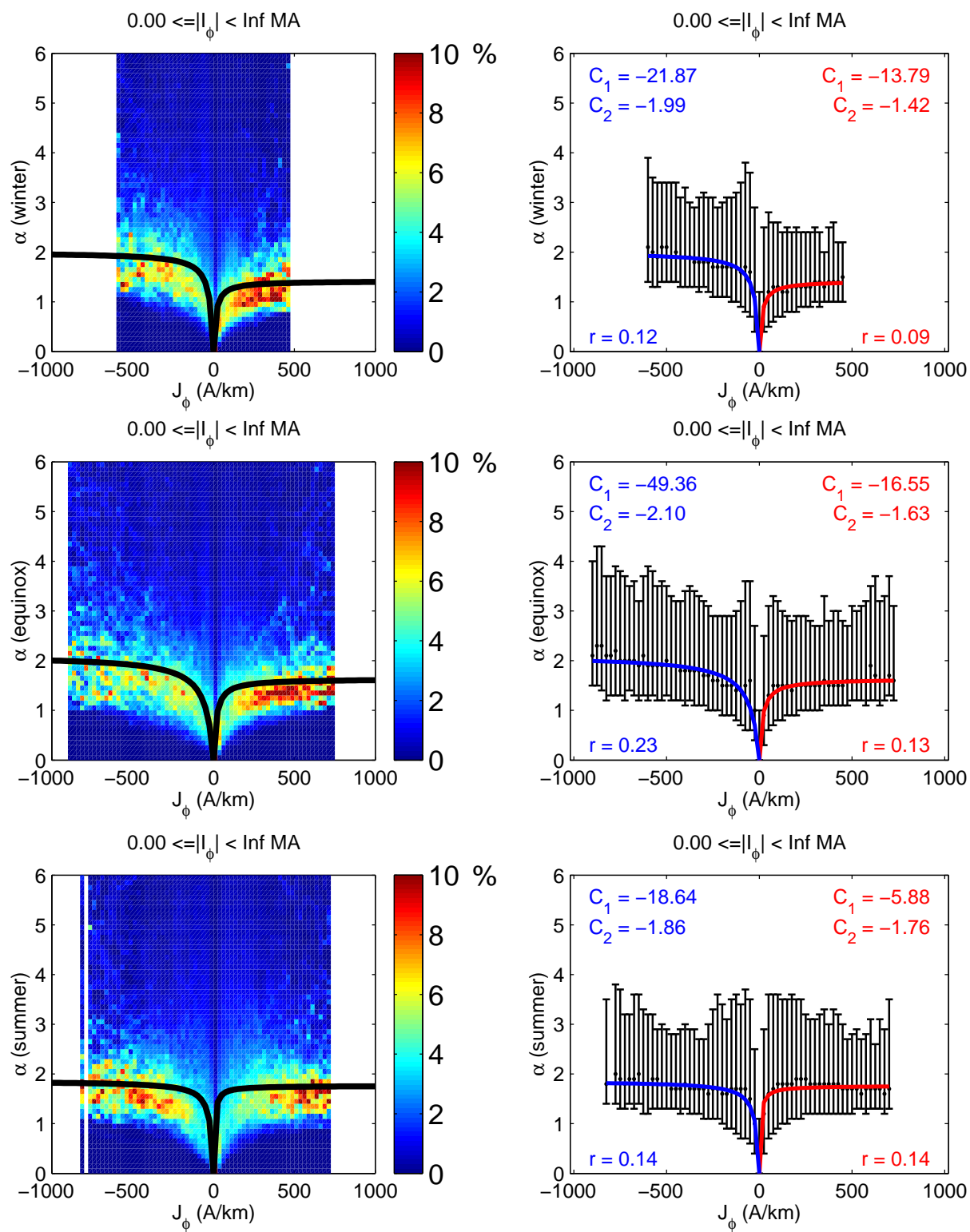

Fig. 14. The same as Fig. 13, except that instead of activity, the data are binned with respect to season: winter (top), equinox (middle) and summer (bottom).

events, during which particle precipitation is higher in the morning sector (Opgenoorth et al., 1994). Towards summer, $\alpha$ becomes higher, especially on the sunlit side of the polar cap. The small scale pattern in the plots prior to midnight may not be entirely reliable due to the relatively small number of data points in that region (see the top left hand side panels of the sets in Fig. 11). Outside the auroral oval, $\alpha$ is quite low in all bins.

\section{Conclusions}

Two years of magnetic data from the CHAMP satellite have been employed to determine statistical estimates for the Hall to Pedersen conductance ratio $\alpha$ as a function of the ionospheric equivalent current density. Under the required 1-D assumption, the equivalent currents determined from ground and satellite magnetic data were shown to be equal, which increased the amount of data available for the statistics by extending it outside ground-based coverage. The conductance ratio was attained from magnetic satellite data by using the 1-D SECS method. This was justified by the assumption 


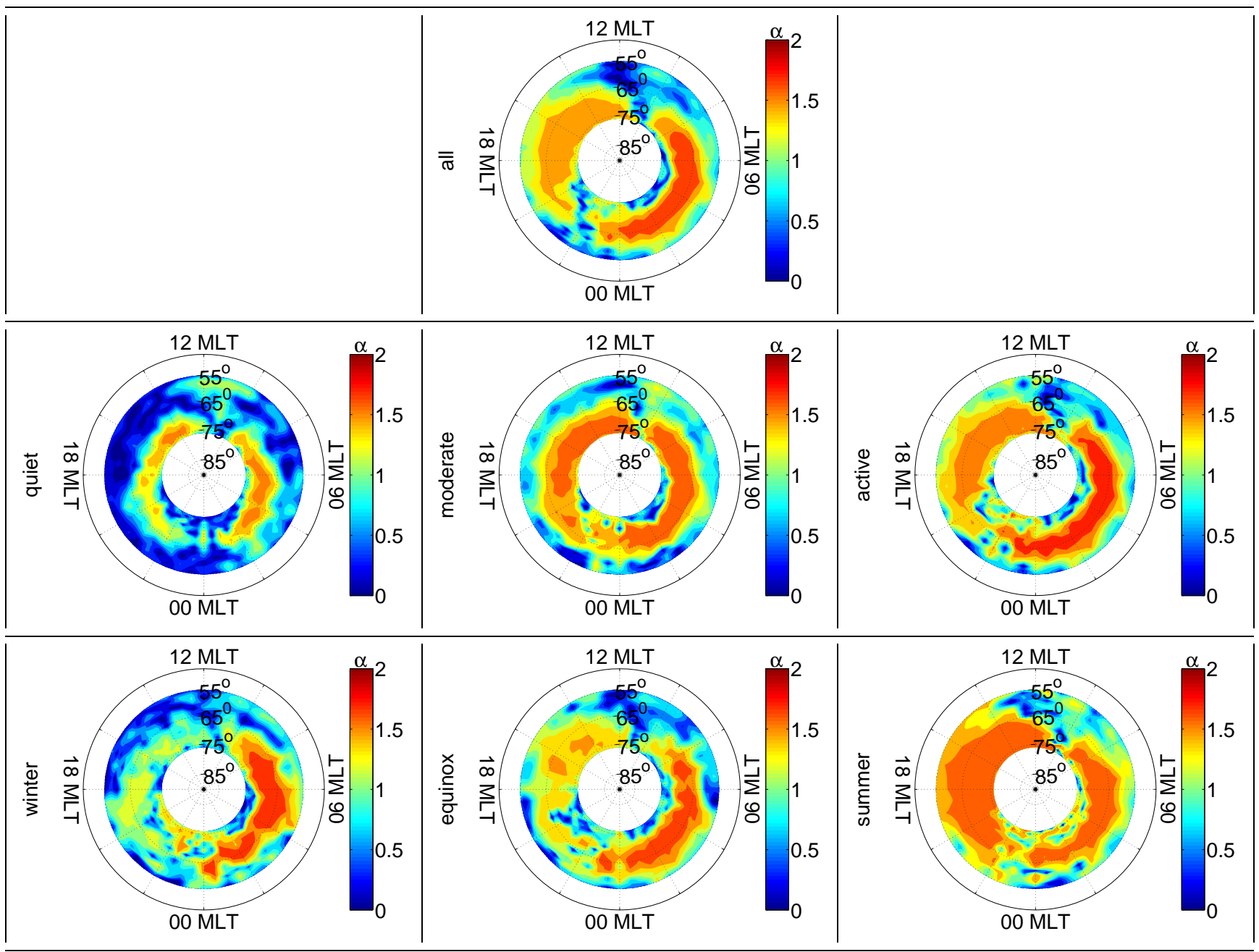

Fig. 15. Average $\alpha$ during 2001-2002 as a function of geomagnetic latitude and MLT. The resolution of the plots is $1^{\circ}$ in latitude and $0.5 \mathrm{~h}$ in MLT, and $\alpha$ is given according to the color bar on the right hand side of each plot. The maps have been determined from the $\phi$ component of the divergence-free current density (cf. $J_{\phi}$ in Figs. 10 and 11) using Eq. (6) with $C_{1}$ and $C_{2}$ from Table 2. The upmost plot is constructed using all available data, the plots in the middle row are binned with respect to activity and those in the bottom row with respect to season (the bin is denoted on the left hand side of each plot).

that in 1-D cases, the ionospheric electric field in $\phi$ direction vanishes. If this is not the case, then, depending of the real electric field direction, $\alpha$ produced by the 1-D SECS method may be too large or too small.

To further justify the 1-D SECS method, $\alpha$ obtained this way was compared with EISCAT measurements. However, there are some problems with such a comparison. One was that we found only five overflights that passed relatively close to the radar with EISCAT data available. None of them passed exactly above EISCAT, with distances varying from 50 to $300 \mathrm{~km}$. If the current distribution really were $1-\mathrm{D}$, this should not be a problem, but in reality the reliability of the currents produced by the 1-D SECS method decreases away from the satellite. To support this, the relative error between $\alpha$ from CHAMP and EISCAT data increased with increasing distance between the satellite and the radar. In two cases out of the total five, however, the two methods gave completely different results. This might be explained by another problem related to this kind of comparison: spatial resolutions of the two methods are different. EISCAT is able to detect structures down to scales of a few kilometers while the resolution of the 1-D SECS method applied to CHAMP data is $\sim 150 \mathrm{~km}$. The radar measures only at a single point, which can understandably produce very different results from the 1-D SECS method. Unfortunately, EISCAT is the only way to obtain direct measurements of $\alpha$ from ground.

Compared to other methods, using magnetic field data from a satellite to determine the conductance ratio ensures 
reliable data over long time sequences. The statistical study was carried out employing over 60001 1-D overpasses of the CHAMP satellite between $55^{\circ}$ and $76.5^{\circ}$ geomagnetic latitude. To get a view of the ionospheric currents during those overflights, maps of all three components were included. A simple relationship (Eq. 6, Table 2) was established between the equivalent current density and $\alpha$ during different condition (activity, season). The relationship between current density and $\alpha$ was observed to be asymmetric for eastward $\left(J_{\phi}>0\right)$ and westward currents $\left(J_{\phi}<0\right)$, or dusk and dawn, and this feature was accommodated by having different coefficients for these two types in Table 2. Since the resulting curves are quite similar for all bins, the only one actually needed is the "all" bin:

$\alpha=\frac{2.07}{\frac{36.54}{\left|J_{\phi}\right|}+1}, \quad J_{\phi}<0$

$\alpha=\frac{1.73}{\frac{14.79}{\left|J_{\phi}\right|}+1}, \quad J_{\phi}>0$,

where the current densities are given in A/ km. Finally as an application, Eq. (6) and Table 2 were used to produce maps of the conductance ratio as a function of geomagnetic latitude and MLT.

Although the data used in establishing the relationship between $\alpha$ and the equivalent current density were required to fulfil the 1-D condition, the same estimates could also be used as the first approximation in 2-D cases. Even in 2-D cases, there is practically always a background electrojet, and $\alpha$ could be estimated from that. A draw-back is that since 1$\mathrm{D}$ cases are not very active, the statistics only apply when the east-west current density does not exceed $1000 \mathrm{~A} / \mathrm{km}$. It should also be borne in mind that since the method is based on statistics, it cannot be applied to get the exact value of $\alpha$ for an event, only the most likely one under the given conditions.

Acknowledgements. The authors wish to thank P. Ritter from GFZ Potsdam for providing the CHAMP satellite data. EISCAT is an international Association supported by Finland (SA), France (CNRS), the Federal Republic of Germany (MPG), Japan (NIPR), Norway (NFR), Sweden (NFR) and the United Kingdom (PPARC). The IMAGE magnetometer data are collected as a Estonian-FinnishGerman-Norwegian-Polish-Russian-Swedish project. The work by L. Juusola was supported by the Finnish Graduate School in Electromagnetics.

Topical Editor M. Pinnock thanks P. Ritter and another referee for their help in evaluating this paper.

\section{References}

Amm, O.: Direct determination of the local ionospheric Hall conductance distribution from two- dimensional electric and magnetic field data: Application of the method using models of typical ionospheric electrodynamic situations, J. Geophys. Res., 100, $21473-21488,1995$.
Amm, O.: Ionospheric elementary current systems in spherical coordinates and their application, J. Geomagn. Geoelectr., 49, 947955, 1997.

Amm, O. and Viljanen, A.: Ionospheric disturbance magnetic field continuation from the ground to the ionosphere using spherical elementary current systems, Earth Planets Space, 51, 431-440, 1999.

Baumjohann, W., Pelunen, R. J., Opgenoorth, H. J., and Nielsen, E.: Joint two-dimensional observations of ground magnetic and ionospheric electric fields associated with auroral zone currents: Current systems associated with local auroral break-ups, Planet. Space Sci., 29, 431-447, 1981.

Davies, J. A. and Lester, M.: The relationship between electric fields, conductances and currents in the high-latitude ionosphere: a statistical study using EISCAT data, Ann. Goephys., 17, 43-52, 1999.

Folkestad, K., Hagfors, T., and Westerlund, S.: EISCAT: An updated description of technical characteristics, Radio Sci., 18, 867-879, 1983.

Fukushima, N.: Generalized theorem for no ground magnetic effect of vertical currents connected with Pedersen currents in the uniform-conductivity ionosphere, Rep. Ionos. Space Res. Japan, 30, 35-40, 1976.

Hedin, A.E.: MSIS-86 thermospheric model, J. Geophys. Res., 92, 4649-4662, 1987.

Iijima, T. and Potemra, T. A.: Field-aligned currents in the dayside cusp observed by TRIAD, J. Geophys. Res., 81, 5971-5979, 1976.

Juusola, L., Amm., O., and Viljanen A.: One-dimensional spherical elementary current systems and their use for determining ionospheric currents from satellite measurements, Earth Planets Space, 58, 667-678, 2006.

Lester, M. and Davies, J. A.: High-latitude Hall and Pedersen conductances during substorm activity in the SUNDIAL-ATLAS campaign, J. Geophys. Res., 101, 26719-26 728, 1996.

Lühr, H., Aylward, A., Buchert, S. C., Pajunpää, A., Pajunpää, K., Holmboe, T., and Zalewski, S. M.: Westward moving dynamic substorm features observed with the IMAGE magnetometer network and other ground-based instruments, Ann. Geophys., 16, 425-440, 1998,

http://www.ann-geophys.net/16/425/1998/.

Olsen, N.: A new tool for determining ionospheric currents from magnetic satellite data, Geophys. Res. Lett., 23, 3635-3638, 1996.

Opgenoorth, H. J., Persson, M. A. L., Pulkkinen, T. I., and Pellinen, R. J.: Recovery phase of magnetospheric substorms and its association with morning-sector aurora, J. Geophys. Res., 99, A3, 4115-4129, 1994.

Papitashvili, V. O., Christiansen, F., and Neubert, T.: A new model of field-aligned currents derived from high-precision satellite magnetic field data, Geophys. Res. Lett., 29(14), doi:10.1029/2001GL014207, 2002.

Press, W. H., Flannery, B. P., Teukolsky, S. A., and Vetterling, W. T.: Numerical Recipes, 2nd ed., pp. 973, Cambridge University Press, Cambridge, 1992.

Pulkkinen, A., Amm, O., Viljanen A., and BEAR Working Group: Ionospheric equivalent current distributions determined with the method of spherical elementary current systems, J. Geophys. Res., 108, 1053-1061, 2003. 
Ritter, P., Lühr, H., Viljanen, A., Amm, O., Pulkkinen A., and Sillanpää, I.: Ionospheric currents estimated simultaneously from CHAMP satellite and IMAGE ground based magnetic field measurements: a statistical study at auroral latitudes, Ann. Geophys., 22, 417-430, 2004, http://www.ann-geophys.net/22/417/2004/.

Robinson, R. M., Vondrak, R. R., Miller, K., Dabbs, T., and Hardy, D.: On calculating ionospheric conductances from the flux and energy of precipitating particles, J. Geophys. Res., 92, 25652569, 1987.

Russell, C. T. and McPherron, R. L.: Semiannual variation of geomagnetic activity, J. Geophys. Res., 78, 92-108, 1973.

Schlegel, K.: Auroral zone E-region conductivities during solar minimum derived from EISCAT data, Ann. Geophys., 6, 129138, 1988,

http://www.ann-geophys.net/6/129/1988/.

Schunk, R. W. and Nagy, A. F.: Ionospheres: physics, plasma physics, and chemistry, Cambridge University Press, Cambridge, 2000 .
Untiedt, J. and Baumjohann, W.: Studies of polar current systems using the IMS Scandinavian magnetometer array, Space Sci. Rev., 63, 245-390, 1993.

Vanhamäki, H., Amm, O., and Viljanen, A.: 1-Dimensional upward continuation of the ground magnetic field disturbance using spherical elementary current systems, Earth Planets Space, 55, 613-625, 2003.

Viljanen, A., Pulkkinen, A., Amm, O., Pirjola, R., Korja, T., and BEAR Working Group: Fast computation of the geoelectric field using the method of elementary current systems and planar Earth models, Ann. Geophys., 22, 101-113, 2004, http://www.ann-geophys.net/22/101/2004/.

Weimer, D. R.: Maps of ionospheric field-aligned currents as a function of the interplanetary magnetic field derived from Dynamics Explorer 2 data, J. Geophys. Res., 106, 12 889-12 902, 2001. 